\title{
Suitability of EST-PCR Markers Developed in Highbush Blueberry for Genetic Fingerprinting and Relationship Studies in Lowbush Blueberry and Related Species
}

\author{
Daniel J. Bell \\ School of Biology and Ecology, University of Maine, Deering Hall, Orono, ME 04473 and U.S. \\ Department of Agriculture, ARS, Henry A. Wallace Beltsville Agricultural Research Center, Genetic \\ Improvement of Fruits and Vegetables Laboratory, 10300 Baltimore Ave., Beltsville, MD 20705
}

Lisa J. Rowland ${ }^{1}$

U.S. Department of Agriculture, ARS, Henry A. Wallace Beltsville Agricultural Research Center, Genetic Improvement of Fruits and Vegetables Laboratory, 10300 Baltimore Ave., Beltsville, MD 20705

\begin{abstract}
James J. Polashock
U.S. Department of Agriculture, ARS, Genetic Improvement of Fruits and Vegetables Laboratory, Blueberry and Cranberry Research Center, 125A Lake Oswego Road, Chatsworth, NJ 08019
\end{abstract}

\author{
Frank A. Drummond \\ School of Biology and Ecology, University of Maine, Deering Hall, Orono, ME 04473
}

\begin{abstract}
AdDITIONAL INDEX WORDs. dendrogram, expressed sequence tags, Vaccinium corymbosum, Vaccinium angustifolium
Abstract. Little is known of the genetic structure and variability of wild fields, or of the dramatic differences in yield among clones (genetic individuals), of lowbush blueberry (Vaccinium angustifolium Ait.), Maine's most economically important fruit crop. Express sequence tag-polymerase chain reaction (EST-PCR) markers that were originally developed for genetic mapping purposes in highbush blueberry (Vaccinium corymbosum L.) are shown here to be valuable for genetic fingerprinting and relationship studies in the related species, $V$. angustifolium. As part of an interspecific genetic relationship study, 14 genotypes, including at least two specimens of each of four closely related Vaccinium L. species (V. pallidum Ait., V. corymbosum, V. boreale Hall \& Aald., and V. myrtilloides Michx.) and the only four pedigreed cultivars of $V$. angustifolium, grouped out as expected in a genetic similarity dendrogram (matrix " $r$ " correlation $=0.91$ ). This work is ultimately aimed at using these markers in exploring how genetic relationship affects yield among proximal and distant breeding individuals via controlled field hand crosses. To help address this issue, a separate group of six individuals of $V$. angustifolium from two managed fields were also genotyped using the EST-PCR markers. The markers were very effective at intraspecific discrimination of individuals within the same field.
\end{abstract}

Lowbush blueberry (Vaccinium angustifolium, section Cyanococcus A. Gray, Ericaceae) is a unique agricultural crop of northeastern North America in that it is wild in origin. Plants are not sown but have been left to reclaim burned and cleared stretches of land over several centuries. In a sense, they have been domesticated not by breeding, but by cultural management practices. It is Maine's most important fruit crop and grows on over 24,000 ha (Yarborough, 1998). Management has focused on optimizing growth conditions, deterring competition from weeds, and minimizing the losses incurred by fungal diseases and insect damage (Yarborough, 1998). These cultural practices have resulted in a 4-fold increase in yield during the last several decades (Yarborough, 2004), but these kinds of increases are unlikely to continue in the absence of detailed

Received for publication 10 June 2008. Accepted for publication 4 Aug. 2008. We would like to thank Dana Robinson, Emmanuel Ajudua, and Elizabeth Ogden for technical assistance. We would also like to thank Drs. Kim Lewers and Mark Ehlenfeldt for their critical reviews of the manuscript.

${ }^{1}$ Corresponding author. E-mail: Jeannine.Rowland@ars.usda.gov. knowledge of the reproductive genetics of this highly polymorphic species.

Lowbush blueberry is a complex tetraploid $(2 \mathrm{n}=4 \mathrm{x}=48)$ proposed to be an autotetraploid of $V$. boreale (Camp, 1945) or an allotetraploid of $V$. boreale $\times V$. pallidum or $V$. boreale $\times V$. myrtilloides (Vander Kloet, 1977, 1978) but with tetrasomic inheritance (Hokanson and Hancock, 1998). It is morphologically and genetically polymorphic (Burgher et al., 2002; Hokanson and Hancock, 1998) and shows greatly varying yields among individual plants. The causes of these differences are unknown. Individual plants reproduce sexually on a 2-year cropping cycle in managed fields and asexually by underground rhizomes. Individual plants can span several meters in diameter, are almost certainly of different ages within fields, and appear as a mosaic patchwork of clones. The species is bee pollinated [rented honeybees (Apis mellifera L.)], which in managed fields with a very high density and acreage of lowbush blueberry clones likely results in a localized pollen distribution based on foraging behaviors and patterns of bees. Thus, a given 
clone is expected to receive significant amounts of its own pollen and that of its near neighbors (F.A. Drummond, personal communications). Native pollinators include more than 100 species of bees, including solitary bees (Andrenidae, Halictidae, Megacilidae) and bumble bees (Bombus Latreille) (Stubbs et al., 1996). In Maine, however, due to the recent decline of native pollinators, rented honeybees are generally stocked at approximately two to four hives per acre to achieve adequate pollination (Drummond, 2002). V. angustifolium is predominantly viewed as self-infertile, requiring outcrossing to achieve good yields (Aalders and Hall, 1961; Hall et al., 1979). A phys- ical separation of male and female function within the same flower (herkogamy) may further enhance outcrossing and reduce pollen discounting (Harder and Wilson, 1998; Nagylaki, 1976).

Although managed intensively, the clones are of wild origin and their spatial genetic structure and relationship patterns are completely unknown. This knowledge is critical to test hypotheses that proximal clones who likely exchange pollen may experience inbreeding depression due to nearness of relationship and thus lower yields. Inbreeding depression has been clearly demonstrated in $V$. angustifolium through pollen chase experiments in which self pollen was "chased" with outcross

Table 1. Summary of Vaccinium corymbosum ESTs used in this study along with their names, accession numbers, sequence length, forward/ reverse primers, putative identifications from BLASTX searches, BLASTX E-values, and annealing temperatures.

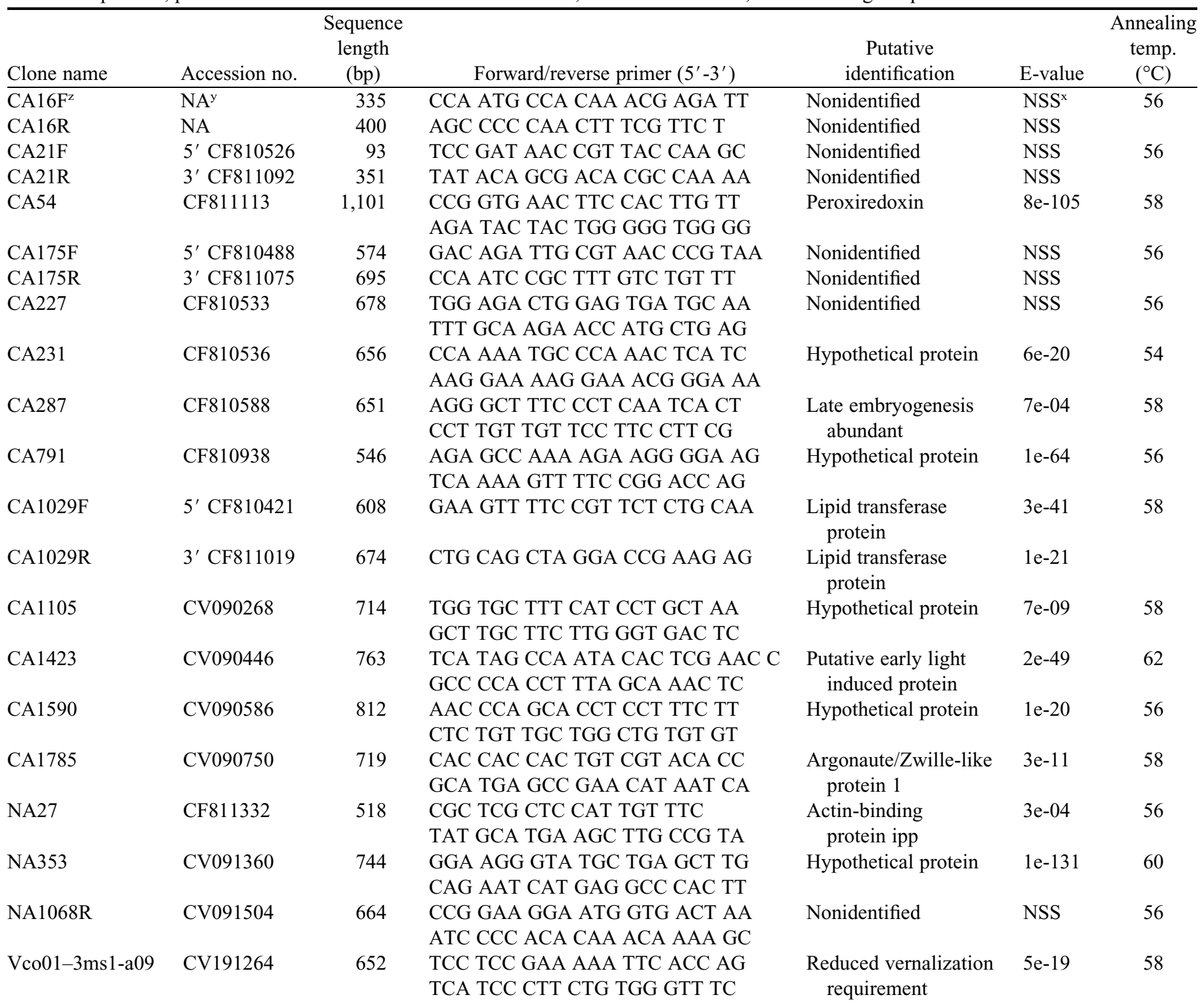

${ }^{\mathrm{z}}$ Most of the cDNAs from the blueberry EST libraries were sequenced only from the $5^{\prime}$ ends, and the PCR primers were designed near the ends of the one available sequence to amplify as large of a portion of the genes as possible. However, a few cDNAs were sequenced from the $5^{\prime}$ and $3^{\prime}$ ends, in which case, the EST clone names end in either an F or an R to indicate the $5^{\prime}$ and $3^{\prime}$ sequence. In those cases, the forward primer was designed from the $5^{\prime}$ end of the $5^{\prime}$ sequence and the reverse primer was designed from the $3^{\prime}$ end of the $3^{\prime}$ sequence, again to amplify as much of the gene as possible from the available sequences.

${ }^{\mathrm{y}} \mathrm{NA}=$ not applicable. The CA16F and CA16R sequences were not deposited in GenBank because they had more than $2 \%$ Ns.

${ }^{\mathrm{x}} \mathrm{NSS}=$ no significant similarity $(P \geq 0.001)$ with a sequence in GenBank. 


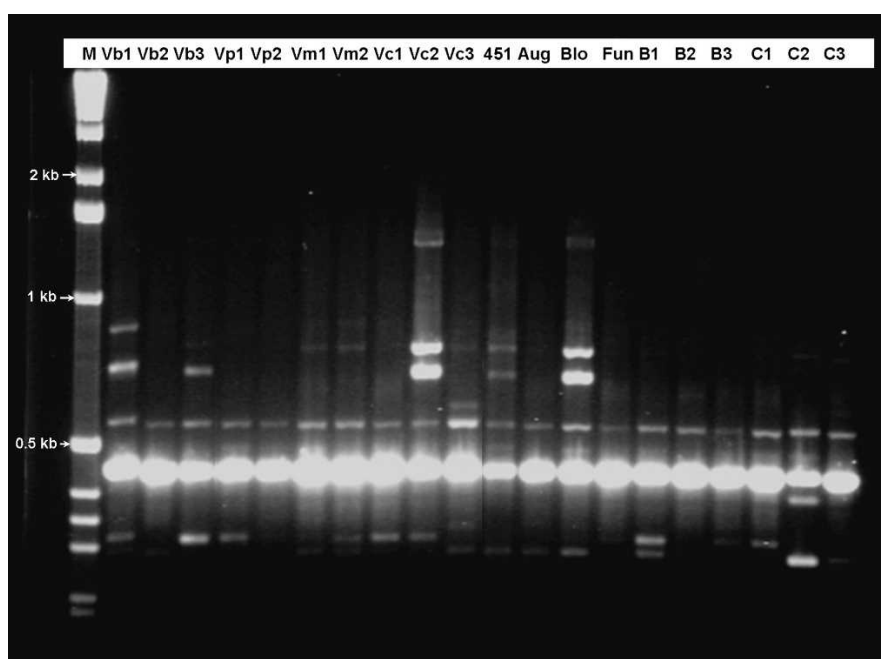

Fig. 1. Agarose gel showing amplification products from Vaccinium corymbosum-derived primer pair NA27 for all 20 Vaccinium genotypes used in this study. The DNA samples were loaded as follows: 1-kb molecular weight ladder from Invitrogen Life Technologies (Carlsbad, CA; M), three $V$. boreale wild selections (Vb1, Vb2, and $\mathrm{Vb} 3$ ), two $V$. pallidum wild selections (Vp1 and Vp2), two $V$. myrtilloides wild selections (Vm1 and $\mathrm{Vm} 2$ ), three $V$. corymbosum wild selections ( $\mathrm{Vc1}, \mathrm{Vc} 2$, and $\mathrm{Vc} 3$ ), four $V$. angustifolium cultivars or named selections ('451', Aug = 'Augusta', Blo = 'Blomidon', and Fun $=$ 'Fundy'), three $V$. angustifolium wild clones from Blueberry Hill Farm (Jonesboro, ME; B1, B2, and B3), and three $V$. angustifolium wild clones from Columbia Farm (Cherryfield, ME; C1, C2, and C3).

pollen after $1 \mathrm{~d}$ and then $3 \mathrm{~d}$ (Hokanson and Hancock, 2000). The increased numbers of aborted seeds in the 3-d treatment versus the 1-d treatment indicates that self pollen does fertilize the ovules but often seeds do not develop properly. This is

Table 2. Homology summary of EST-PCR products that were cloned and sequenced from Vaccinium angustifolium, including the primers and genotypes that were used, sizes of fragments in bps, genetic variation observed among genotypes, and whether the PCR products were homologous to the original Vaccinium corymbosum cDNAs from which the primer sequences were derived.

\begin{tabular}{llccc}
\hline $\begin{array}{l}\text { EST-PCR } \\
\text { primer pair }\end{array}$ & Genotype & $\begin{array}{c}\text { Approximate size } \\
\text { of fragment (bp) }\end{array}$ & $\begin{array}{c}\text { Monomorphic (M) } \\
\text { or polymorphic (P) }\end{array}$ & $\begin{array}{c}\text { Homologous } \\
\text { to cDNA }\end{array}$ \\
\hline CA54 & 'Augusta' & 1,800 & $\mathrm{M}$ & No \\
CA227 & B1 & 270 & $\mathrm{P}$ & No \\
& $\mathrm{B} 1$ & 425 & $\mathrm{P}$ & Yes \\
CA231 & $\mathrm{B} 1$ & 500 & $\mathrm{M}$ & Yes \\
& $\mathrm{B} 3$ & 325 & $\mathrm{M}$ & No \\
CA287 & $\mathrm{B} 3$ & 575 & $\mathrm{M}$ & No \\
& $\mathrm{B} 3$ & 975 & $\mathrm{P}$ & Yes \\
CA1029 & $\mathrm{B} 3$ & 1,020 & $\mathrm{M}$ & Yes \\
CA1590 & $\mathrm{B} 3$ & 1,200 & $\mathrm{M}$ & Yes \\
& $\mathrm{B} 3$ & 650 & $\mathrm{P}$ & No \\
& $\mathrm{B} 3$ & 725 & $\mathrm{P}$ & Yes \\
CA1785 & B3 & 800 & $\mathrm{P}$ & No \\
& 'Augusta' & 320 & $\mathrm{P}$ & Yes \\
& 'Fundy' & 450 & $\mathrm{P}$ & Yes \\
CA1807 & 'Blomidon' & 1,400 & $\mathrm{P}$ & Yes \\
NA27 & B3 & 1,200 & $\mathrm{M}$ & Yes \\
& 'Blomidon' & 450 & $\mathrm{M}$ & Yes \\
Vco01-3ms1-a09 & 'Blomidon' & 700 & $\mathrm{P}$ & Yes \\
& B1 & 1,250 & $\mathrm{P}$ & Yes \\
\hline
\end{tabular}

interpreted as early-acting inbreeding depression (Hokanson and Hancock, 2000) and has therefore been hypothesized as a possible cause of the depression of yields in patches within fields comprised of closely related individuals (Myra et al., 2004). The identification of molecular markers that are informative across multiple and varied blueberry genotypes would allow future testing of the inbreeding hypothesis by characterizing relatedness among participants in controlled field crosses.

Rowland et al. (2003b) developed express sequence tagpolymerase chain reaction (EST-PCR) markers from EST libraries derived from floral buds of cold acclimated and nonacclimated highbush blueberry plants ( $V$. corymbosum). Surprisingly, $\approx 50 \%$ of the primers designed from the ends of the ESTs amplified polymorphic fragments among the tested genotypes that were detectable directly on agarose gels and thus represented scoreable length polymorphisms. This high level of polymorphism was explained as likely due to blueberry species being primarily outcrossing and, thus, highly heterozygous. The Cyanococcus taxon has been considered a very difficult one from a systematics perspective throughout the 20th century due to "intermittent but strong gene flow" (Vander Kloet, 1973) among populations in the section and the prevalence of polyploidy (Lyrene et al., 2003; Vander Kloet, 1978). Until now, the only molecular markers used in lowbush blueberry have been RAPD markers, which are anonymous markers amplified from short random primers, 10 bases in length (Burgher et al., 2002). Although EST technology is generally viewed first as a gene discovery tool for expression profiling, the development and use of EST-PCR products for phylogenetics and studies of intrapopulation genetic variation have been recently shown to be effective in other taxa such as Rhododendron L. (Wei et al., 2006) and Picea abies L. (Schubert et al., 2001).

For a variety of reasons, including better reproducibility than RAPDs and derivation from inferred coding regions, we asked whether we could reliably import these EST-PCR markers originally developed for highbush blueberry to fingerprint and estimate genetic similarity among clones within fields of lowbush blueberry. The study described here was designed to test whether these markers would reliably discriminate among five related species of blueberry that are represented in Maine and, in particular, whether they would be useful for estimating intraspecific variation within the wild, commercial $V$. angustifolium.

\section{Materials and Methods}

Plant material. Genotypes for the interspecific study included wild selections of three diploid $V$. boreale [one from Cadillac Mountain, Mount Desert Island, ME, and two from Nova Scotia that we obtained from N. Vorsa (Marucci Center for Blueberry and Cranberry Research and Extension, Chatsworth, NJ)], two diploid $V$. pallidum (Chatsworth, $\mathrm{NJ}$ ), two diploid $V$. myrtilloides (Jonesboro, ME), three tetraploid $V$. corymbosum (Winterport, ME), and four tetraploid cultivars of 
$V$. angustifolium of known pedigree that were generated from a breeding program in the $1970 \mathrm{~s}$ in Canada (451, Augusta, Blomidon, and Fundy). 'Blomidon' is the progeny of ' 451 ' $\times$ 'Augusta' and 'Fundy' is the open-pollinated progeny of 'Augusta', therefore, the half-sibling of 'Blomidon'. To determine usefulness of EST-PCR markers for intraspecific discrimination of clones within the same or near fields, six clones of $V$. angustifolium were selected from two commercially managed fields (three clones from Blueberry Hill Farm in Jonesboro, $\mathrm{ME}$, and three clones from Columbia Farm in Cherryfield, ME). These two fields were chosen because they have been the ongoing sites for 3 years (2005-08) of field data collections, including natural yield measurements, phenological data, and field experiments using controlled hand crosses.

Genomic DNA extraction AND PCR CONDITIONS. DNA extractions from leaf tissue, primer design from ESTs, PCR amplification, and gel electrophoresis conditions were performed as described previously (Rowland et al., 2003b). Twenty-four primer pairs were selected for testing from a subset known to identify polymorphisms in highbush blueberry. Gels were stained with ethidium bromide, and markers were visualized under ultraviolet light and digitally photographed. Bands were scored as dominant markers, either (1) present or (0) absent. All reactions were run at least twice. Only clear, reproducible bands that ranged from $\approx 300$ to $2500 \mathrm{bp}$ were scored. The resulting data of genotypes and the presence/ absence of scored bands provided the binary data grid that was statistically analyzed. Also, to provide additional evidence of the fingerprinting integrity of these markers, DNA was isolated in separate extractions, and run in separate PCR reactions from two ramets connected to a single rhizome (genet) from a randomly chosen clone at Blueberry Hill (Jonesboro, ME). This was seen as a control test, barring somatic mutations, to assure that the two ramet samples matched perfectly for every primer pair chosen.

Cloning and Sequencing of Selected est-PCR PRoducts. A selection of EST-PCR amplicons, including monomorphic and polymorphic fragments, were excised from gels, cloned, and sequenced as described previously (Rowland

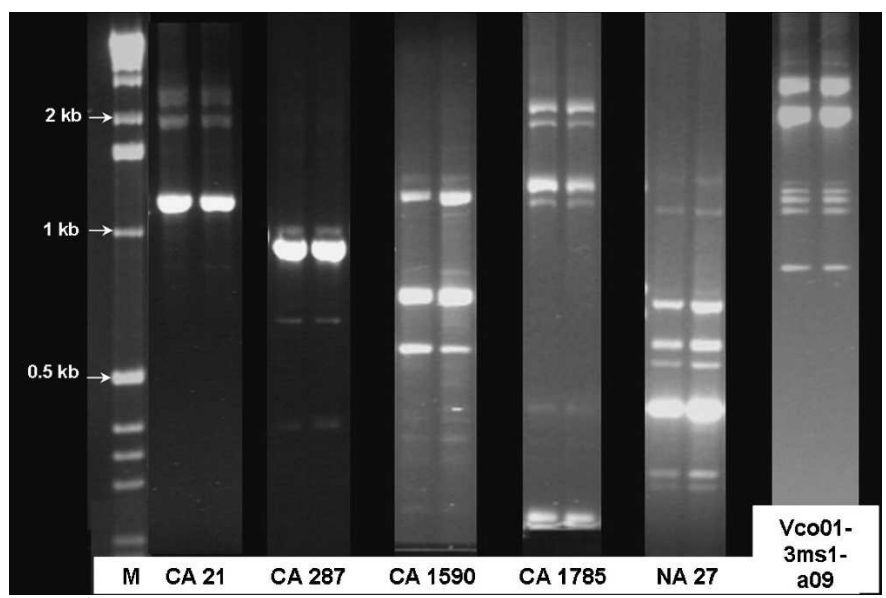

Fig. 2. Agarose gel showing amplification products from template DNA isolated in separate duplicate extractions, and run in separate duplicate PCRs from two ramets connected to a single rhizome (genet) from a randomly chosen Vaccinium angustifolium clone at Blueberry Hill Farm (Jonesboro, ME) using primer pairs CA21, CA287, CA1590, CA1785, NA27, and Vco01-3ms1-a09. et al., 2003a). Using BLAST (Altschul et al., 1990) algorithms, sequence similarity of the PCR fragments to the original cDNAs from which the primers were designed was determined.

Genetic Relationship analysis. Of the 24 primer pairs tested, 17 were chosen for the visual clarity of the separated amplified fragments and because they produced length polymorphisms among the genotypes. The Numerical Taxonomy and Multivariate Analysis System (NTSYS-PC, version 2.2; Exeter Software, Setauket, NY) (Rohlf, 2002) was used to generate a pairwise similarity matrix using the Dice coefficient function (Dice, 1945) from the binary data. For clustering and dendrogram construction, we performed an unweighted pairgroup method (UPGMA) and a neighbor-joining cluster analysis from the similarity matrix. A confidence control of the clusters was performed by generating a cophenetic matrix from the tree and comparing the cophenetic matrix to the original similarity matrix via a Mantel test (Mantel, 1967). This test gives a confidence estimate for how well the distance relationships are preserved between the two matrices (Sokal, 1979). A higher " $r$ " value indicates a higher confidence in the clustering. Finally, as a further aid in partitioning within and among species genetic variation, particularly among clone variation of $V$. angustifolium, an analysis of molecular variance (AMOVA) was carried out using GenAlex, version 6.1 (Peakall and Smouse, 2006), using the five species groups as "populations."

\section{Results and Discussion}

EST-PCR MARKERS FOR DETECTING INTERSPECIFIC GENETIC VARIATION. EST-PCR markers were first tested on a group of 14 genotypes to determine if they could distinguish among several Vaccinium species and be useful in assessing genetic relationships within the $V$. angustifolium species. At least two specimens each of five species of the genus Vaccinium ( $V$. angustifolium, $V$. boreale, $V$. corymbosum, $V$. myrtilloides, and $V$. pallidum), which have been described taxonomically as being closely related (Vander Kloet, 1983), were collected. For $V$. angustifolium, four cultivars from the only known pedigree group (see Materials and Methods) in this species were used. Table 1 presents a summary of the EST-PCR primer combinations that were deemed useful. In all, 17 primer pairs were chosen from the original 24 that were tested, yielding a total of 81 polymorphic, scorable markers ( $\approx 4.8$ bands per primer). The number of bands per primer is not surprising considering that most genes are members of small multigene families and that two of the species used in this study, $V$. angustifolium and $V$. corymbosum, are tetraploids. All 17 primer pairs resulted in successful amplification in all the Vaccinium species tested. A gel of the amplification products from one primer pair NA27 (from a cDNA clone encoding a putative actin-binding protein) is shown in Fig. 1. The seven primer pairs that were not used from the 24 tested were either monomorphic across all genotypes, did not amplify well, were faint, or otherwise were not reliably reproducible. Of the 81 polymorphic markers, 35 were present in the $V$. boreale selections, 19 in $V$. pallidum, 29 in $V$. myrtilloides, 35 in $V$. corymbosum, and 46 in the $V$. angustifolium selections.

EST-PCR MARKERS FOR DETECTING INTRASPECIFIC GENETIC VARIATION. These 17 primer pairs were also tested on six wild clones of $V$. angustifolium selected from two managed fields (three from each field) in Maine to determine their effectiveness 
at intraspecific discrimination of clones within the same or near fields. Only one of the 17 primer pairs, CA1029, did not yield any scoreable, polymorphic markers among this group of genotypes. The other 16 primer pairs yielded a total of 53 scoreable polymorphic markers.

Sequencing anAlysis of ESTPCR Products. We cloned and sequenced a subset of 20 ESTPCR fragments from the $V$. angustifolium genotypes, about equally divided between polymorphic and monomorphic fragments, resulting from 10 different EST primer pairs (Table 2). We considered this a sufficient number to give a good indication of whether the amplified fragments were generally similar in sequence to the highbush blueberry cDNAs from which the primer sequences were derived. All of the sequenced fragments had homology to the primer sequences at the ends. Fourteen of the 20 fragments $(70 \%)$ had high homology to the original cDNA inserts from which the primer pairs were designed, while the other six had low homology or no significant similarity. In the original work describing the development of the EST-PCR markers from $V$. corymbosum sequences (Rowland et al., 2003b), seven of nine fragments $(77 \%)$ cloned from $V$. corymbosum genotypes had homology to the cDNAs from which they were derived, which is on this same order of magnitude. Given the likelihood that many of these cDNAs represent genes that are members of multigene families and that occasionally primers may be designed in highly repeated motifs, this seems reasonable. Furthermore, because scoring was conservative and the bands were reproducible, then regardless of homology, they should represent true genetic differences and therefore be useful in genetic relationship studies. Finally, for every primer pair that resulted in successful amplification, the ramet/ramet PCR profile comparison within a genet was identical, indicating that the fingerprinting capabilities of these markers are extremely robust (Fig. 2).

Clustering relationships and Dendrogram anAlysis. A dendrogram based on the UPGMA clustering analysis of the 81 loci from the interspecific study is shown in Fig. 3. The Mantel test indicated an excellent fit (Sokal, 1979) of the similarity

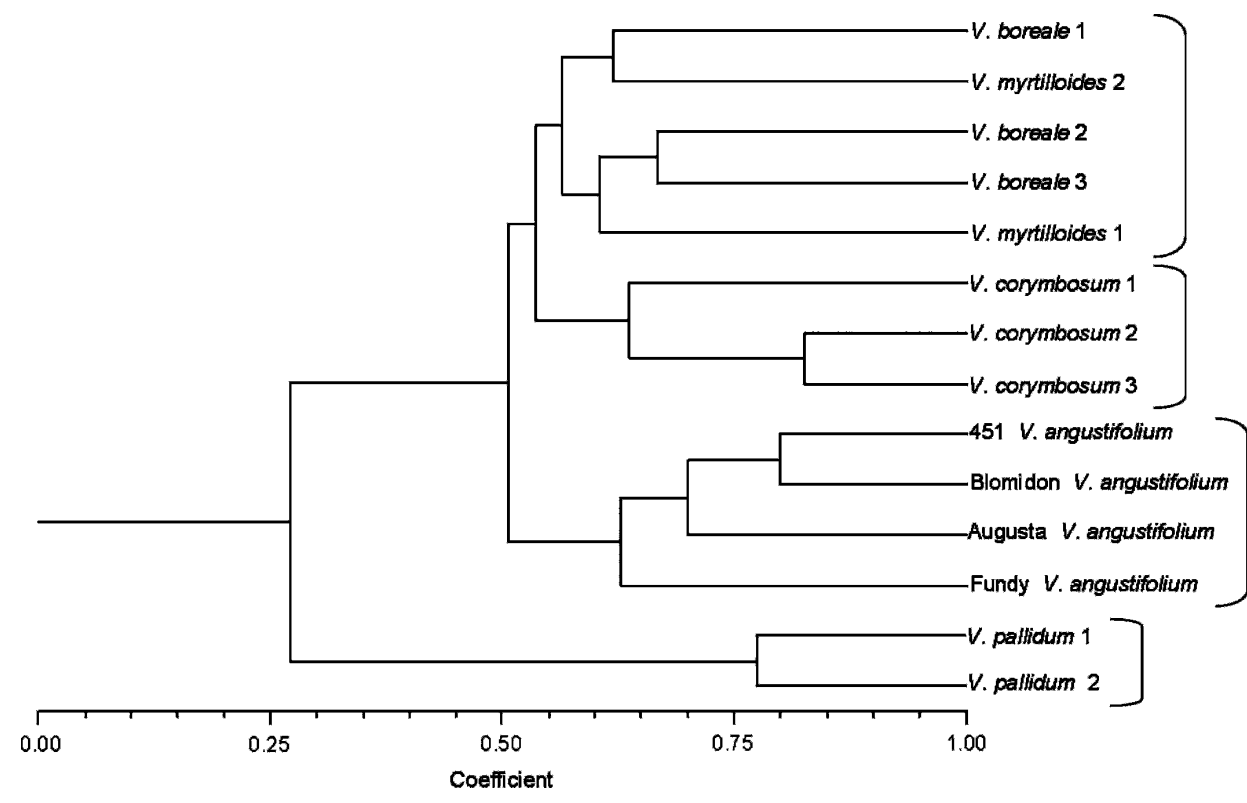

Fig. 3. Dendrogram from a UPGMA clustering analysis depicting groupings and similarity relationships among the 14 Vaccinium genotypes (of $V$. angustifolium, $V$. boreale, $V$. corymbosum, $V$. myrtilloides, and $V$. pallidum) in the interspecific panel study. The calculated goodness of fit " $r$ " from the Mantel test run in NTSYS-PC (version 2.2; Exeter Software, Setauket, NY) was 0.91.

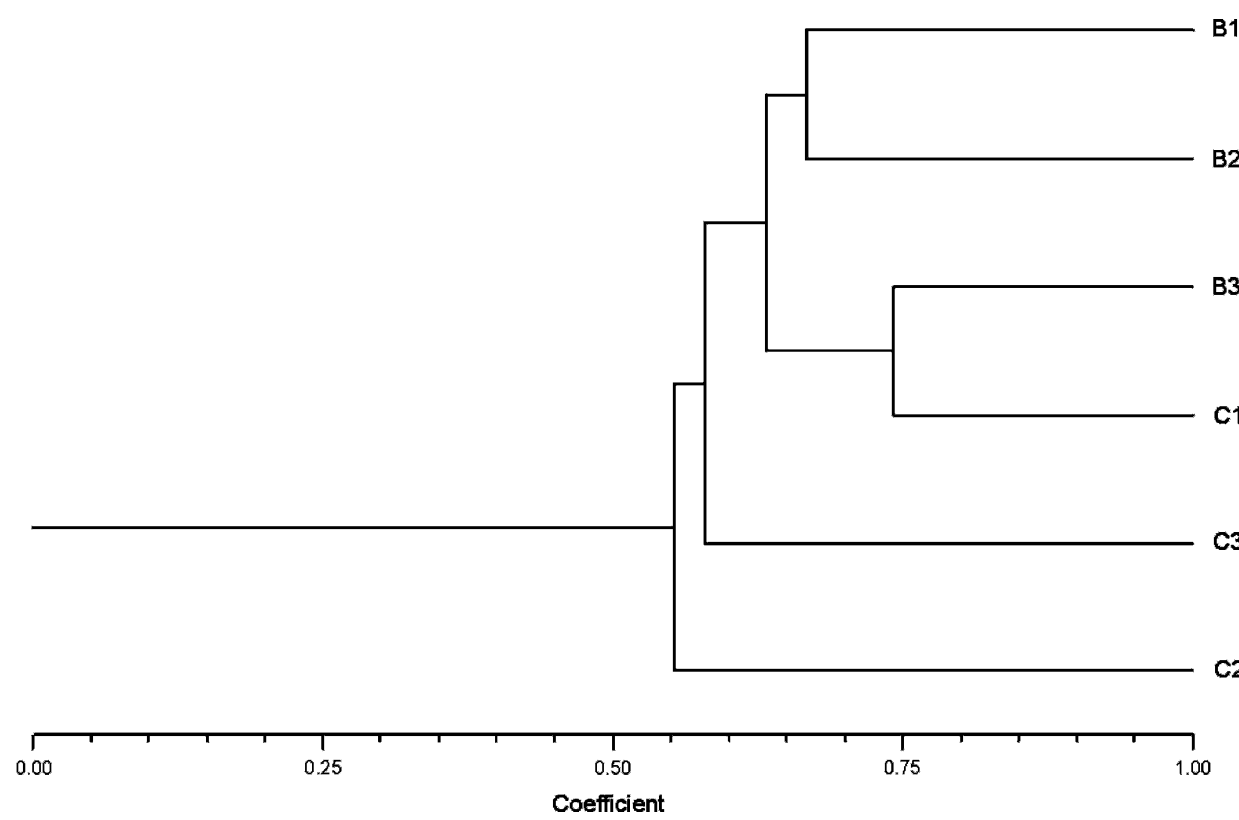

Fig. 4. Dendrogram from a UPGMA clustering analysis depicting the intraspecific similarity relationships among the six Vaccinium angustifolium clones from the same or nearby fields. The calculated goodness of fit " $r$ " from the Mantel test was 0.78. "B" indicates clones from Blueberry Hill Farm (Jonesboro, ME) and " $\mathrm{C}$ " clones from Columbia Farm (Cherryfield, ME; $\approx 12 \mathrm{~km}$ from Blueberry Hill Farm).

matrix to the tree (" $r$ " matrix correlation value $=0.91$ ). The groupings were as expected showing four clades: 1) the $V$. pallidum selections, 2) the $V$. angustifolium cultivars, 3) the $V$. corymbosum selections, and 4) a $V$. boreale/myrtilloides selection complex. The mixed grouping of the $V$. boreale/ myrtilloides representatives is consistent with a previous isozyme study (Bruederle and Vorsa, 1994) that showed that these two species are very closely related, sharing a unique combination of alleles at five isozyme loci. We would expect that the 
inclusion of additional primer combinations would help resolve more clearly these two closely related species. However, interspecific hybridization among homoploids in section Cyanococcus occurs readily ( $V$. boreale and $V$. myrtilloides are diploids) and may present problems in unambiguously resolving these two species if a hybrid complex is involved. Within the $V$. corymbosum group, it is interesting to note that the $V$. $\operatorname{cor} 2$ and $V$. cor 3 samples that were collected within $100 \mathrm{~m}$ of each other grouped closer together than did the third $V$. cor 1 sample that was collected several kilometers away. Also, within the $V$. angustifolium group, 'Blomidon' grouped closer to its parents ('451' and 'Augusta') than it did to its half-sibling ('Fundy'), as would be expected. The tree resulting from the neighbor-joining analysis was virtually identical to the UPGMA results in the interspecific and intraspecific groupings, thus it is not shown.

A second UPGMA dendrogram (Fig. 4) is shown for the two groups of three individuals of $V$. angustifolium from two fields near Jonesboro, ME, which are $\approx 12 \mathrm{~km}$ apart. This dendrogram was based on 53 markers that were polymorphic among these genotypes, a subset of the 81 that were scored in the crossspecies group above. Considered alone, this dendrogram had a fair goodness of fit by the Mantel test of " $r$ " $=0.78$, because of the lower number of polymorphic markers. It is clear from this dendrogram that all clones could be distinguished from each other, with the highest similarity value between clones being $\approx 0.74$. It is consistent with expectations that the range of similarity of the intraspecific group was much tighter at 0.55 to 0.74 in comparison with the cross-species range of 0.27 to 0.83 . In addition, the upper range of similarity $(0.74)$ between clones $\mathrm{B} 1$ and $\mathrm{C} 3$, each from a separate field, reflect what might be approaching the highest level of relationship possible because this range approximates the similarity of parents to offspring (Fig. 3 ), which ranged from $\approx 0.70$ to 0.80 . Although the overall range of similarity tightened in the intraspecific $V$. angustifolium group compared with the interspecific range, an AMOVA revealed two features of all five groups studied: 1) that all five species, when treated as populations in AMOVA, formed statistically significant groups with a total among variation of $37 \%[P($ rand $\geq$ data $)=0.010]$ and 2$) 63 \%$ of the total variation was attributed to within group (species) variation, i.e., among genotypes within that species. Of particular note, $V$. angustifolium showed almost 1.5 times more within population variation than the next highest group, the $V$. boreale. We consider this important because this clearly demonstrates the ability of these markers to genetically differentiate intraspecific clonal variation within our experimental crop.

RAPD markers have been used in recent studies on other rhizomatous Vaccinium species to distinguish among clones or patches. Persson and Gustavsson (2001) found that 43 RAPD markers could discriminate 29 unique genetic individuals from 129 total samples taken of lingonberry (Vaccinium vitis-idaea L.). Kreher and Collins (2000) showed that 15 markers distinguished 67 genets of 99 total samples of deerberry (Vaccinium stamineum L.). Here, we were able to use as few as 28 markers from six primers to uniquely distinguish all the 10 lowbush blueberry clones in this study. Thus, both RAPDs and our EST-PCR markers are efficient at identifying genets within clonal populations.

ConCLUding REMARKs. From this work, we conclude that the EST-PCR markers are useful for all the species tested, resulting in strong, reproducible polymorphic amplification products. In addition, the EST-PCR markers were very effective at estimating genetic relationships, in agreement with pedigree information, and at distinguishing even closely spaced $V$. angustifolium clones within the same field (range Blueberry Hill Farm: 89.1167.2 m; Columbia Farm: 17.3-33.2 m). In conclusion, these markers should prove highly useful in future research on genetic structure in wild fields, in testing hypotheses regarding yield variation as a function of genetic similarity, and for phylogenetic analysis among species of Vaccinium.

\section{Literature Cited}

Aalders, L.E. and I.V. Hall. 1961. Pollen incompatibility and fruit set in lowbush blueberries. Can. J. Genet. Cytol. 3:300-307.

Altschul, S.F., W. Gish, W. Miller, E.W. Myers, and D.J. Lipman. 1990. Basic local alignment search tool. J. Mol. Biol. 215:403410.

Bruederle, L.P. and N. Vorsa. 1994. Genetic differentiation of diploid blueberry, Vaccinium sect. Cyanococcus (Ericaceae). Syst. Bot. 19:337-349.

Burgher, K.L., A.R. Jamieson, and X. Lu. 2002. Genetic relationships among lowbush blueberry genotypes as determined by randomly amplified polymorphic DNA analysis. J. Amer. Soc. Hort. Sci. 127:98-103.

Camp, W.H. 1945. The North American blueberries with notes on other groups of Vacciniaceae. Brittonia 25:203-275.

Dice, L.R. 1945. Measures of the amount of ecological association between species. Ecology 26:297-302.

Drummond, F.A. 2002. Honey bees and blueberry pollination. Univ. Maine Coop. Ext. Fact Sheet No. 629. Univ. Maine Ext. No 2079.

Hall, I.V., L.E. Aalders, N.L. Nickerson, and S.P. Vander Kloet. 1979. The biological flora of Canada. 1. Vaccinium angustifolium Ait., sweet lowbush blueberry. Can. Field-Naturalist 93:415-430.

Harder, L.D. and W.G. Wilson. 1998. A clarification of pollen discounting and its joint effects with inbreeding depression on mating system evolution. Amer. Naturalist 152:684-695.

Hokanson, K. and J. Hancock. 1998. Levels of allozymic diversity in diploid and tetraploid Vaccinium sect. Cyanococcus (blueberries). Can. J. Plant Sci. 78:327-332.

Hokanson, K. and J. Hancock. 2000. Early-acting inbreeding depression in three species of Vaccinium (Ericaceae). Sex. Plant Reprod. 13:145-150.

Kreher, S.A. and B.S. Collins. 2000. Genetic variation within and among patches of the clonal species, Vaccinium stamineum L. Mol. Ecol. 9:1247-1252.

Lyrene, P.M., N. Vorsa, and J.R. Ballington. 2003. Polyploidy and sexual polyploidization in the genus Vaccinium. Euphytica 133:2736.

Mantel, N. 1967. The detection of disease clustering and a generalized regression approach. Cancer Res. 27:209-220.

Myra, M., K. MacKenzie, and S.P. Vander Kloet. 2004. Investigation of a possible sexual function specialization in the lowbush blueberry (Vaccinium angustifolium Aiton. Ericaceae). Small Fruits Rev. 3:313-324.

Nagylaki, T. 1976. A model for the evolution of self-fertilization and vegetative reproduction. J. Theor. Biol. 58:55-58.

Peakall, R. and P.E. Smouse. 2006. GENALEX 6: Genetic analysis in Excel. Population genetic software for teaching and research. Mol. Ecol. Notes 6:288-295.

Persson, H.A. and B.A. Gustavsson. 2001. The extent of clonality and genetic diversity in lingonberry (Vaccinium vitis-idaea L.) revealed by RAPDs and leaf-shape analysis. Mol. Ecol. 10:1385-1397.

Rohlf, F.J. 2002. NTSYS-PC, vers. 2.2. Exeter Software, Setauket, NY. Rowland, L.J., A.L. Dhanaraj, J.J. Polashock, and R. Arora. 2003a. Utility of blueberry-derived EST-PCR primers in related Ericaceae species. HortScience 38:1428-1432.

Rowland, L.J., S. Mehra, A. Dhanaraj, E.L. Ogden, J.P. Slovin, and M.K. Ehlenfeldt. 2003b. Development of EST-PCR markers for 
DNA fingerprinting and genetic relationship studies in blueberry (Vaccinium, section Cyanococcus). J. Amer. Soc. Hort. Sci. 128:682-690.

Schubert, R., G. Mueller-Starck, and R. Riegel. 2001. Development of EST-PCR markers and monitoring the intrapopulational genetic variation in Picea abies (L.). Karst. Theor. Appl. Genet. 103:1223-1231.

Sokal, R.R. 1979. Testing statistical significance of geographic variation patterns. Syst. Zool. 28:227-231.

Stubbs, C.S., E.A. Osgood, J.B. Dimond, and F.A. Drummond. 1996. Hymenoptera diversity in Maine, p. 81-86. In: S.C. Gawler, J.J. Albright, P.D. Vickery, and F.C. Smith (eds.). Biological diversity in Maine. Maine Natural Areas Program, Maine Forest Biodiversity Project, Augusta, ME.

Vander Kloet, S.P. 1973. The North American blueberries revisited: A taxonomic study of Vaccinium section Cyanococcus Gray, Queen's University at Kingston, Ontario, Canada. PhD Diss.
Vander Kloet, S.P. 1977. The taxonomic status of Vaccinium boreale. Can. J. Bot. 55:281-288.

Vander Kloet, S.P. 1978. Systematics, distribution, and nomenclature of the polymorphic Vaccinium angustifolium. Rhodora 80:358376.

Vander Kloet, S.P. 1983. The taxonomy of Vaccinium section Cyanococcus: A summation. Can. J. Bot. 61:256-266.

Wei, H., Y. Fu, and R. Arora. 2006. Utilization of intron-flanking ESTspecific markers in the phylogenetic analysis and parentage identification of rhododendron species and hybrids. J. Amer. Soc. Hort. Sci. 131:814-819.

Yarborough, D. 1998. Wild blueberry fact sheet: Wild blueberry culture in Maine. Univ. Maine Coop. Ext. Fact Sheet No. 220.

Yarborough, D. 2004. Factors contributing to the increase in productivity in the wild blueberry industry. Small Fruits Rev. 3:3343. 